\title{
EXTREME HIGH VACUUM TECHNOLOGY FOR PARTICLE ACCELERATORS
}

\author{
C. Benvenuti, CERN, Geneva, Switzerland
}

\begin{abstract}
Linearly distributed pumping is highly desirable for Storage Rings to limit the pressure rise due to the outgassing induced by the circulating beams. As a byproduct, linear pumping allows extremely low pressures (down to the $10^{-14}$ Torr range) to be achieved in static vacuum conditions.

Non Evaporable Getters (NEGs), in the form either of a strip as adopted for LEP or of a thin film coating, may provide linear pumping in a simple and economic way. Compared to the strip, thin film coatings present the additional benefits of reduced beam-induced and thermal outgassing, larger pumping surface, lower activation temperature and applicability to vacuum chambers of very small aperture. The most recent developments of this technique and the present XHV measuring capabilities will be described and discussed with the help of some examples.
\end{abstract}

\section{INTRODUCTION}

Although all accelerators need vacuum to keep the particles on the desired trajectories, ultra-high vacuum conditions are mandatory only for Storage Rings, for which beam life-times in excess of $10 \mathrm{~h}$ are usually required.

The first machine where extremely low pressures (XHV, below $10^{-12}$ Torr) were achieved to limit both beam neutralization and the beam induced pressure rise [1], was the CERN Intersecting Storage Rings (ISR). The disturbing feature of this latter effect is that the presence of one single weak point may result in a local pressure run-away and beam loss. To counteract this catastrophic event, very clean vacuum chamber walls are required, possibly combined with large pumping speeds. Both these remedies have been successfully adopted for the ISR. All vacuum chambers have undergone argon-oxygen discharge cleaning and Titanium Sublimation pumps have been massively added. Considering the vacuum chamber limited conductance (which however was fortunately quite large for the ISR if compared to that of the present machines) a linearly distributed pumping would have been highly desirable, but this solution did not exist at that time.

Linear pumping is also desirable for electron storage rings although for different reasons. The difficulty here consists in keeping the average pressure low, in spite of the large gas desorption induced by synchrotron radiation. Fortunately the outgassing decreases during machine operation, and eventually low enough pressures may be achieved. However, applying large pumping speeds allows the conditioning time, during which the machine design performance is not reached, to be reduced.
Linear pumping was adopted for the first time for electron machines [2] in the form of Sputter-Ion pumps making use of the magnetic field of the dipole bending magnets. Although very successful, this solution presents the major inconvenience that no pumping is available if the magnetic field is too weak or absent.

Mainly for this reason, a linear Non-Evaporable Getter (NEG) pump was proposed [3] and adopted for the Large Electron Positron Collider (LEP) at CERN [4]. In this machine the main pumping is provided by about $23 \mathrm{~km}$ of NEG strip (see Section 3 of this report), with a marginal complement of appendage Sputter-Ion pumps, required for gases such as methane or argon which NEGs do not pump.

Both the integrated Sputter-Ion and the NEG strip pumps must be electrically isolated from the vacuum chamber and powered via feedthroughs. They are housed in a lateral extension of the vacuum chamber, which must be large enough to guarantee safe operating conditions against short circuits. Therefore they are space demanding and result in a more complex and sometimes convoluted vacuum chamber design.

A more convenient approach consists in using a low activation temperature NEG strip, which may be passively activated during the standard bakeout process [5]. In this case, no electrical constraints exist and more strip may be inserted in a chamber of reduced dimensions. However, even the lowest activation temperature of the existing NEG strips $\left(350{ }^{\circ} \mathrm{C}\right)$ proscribes the use of copper and aluminum which are the standard materials for the vacuum chamber construction of electron machines, because of their good thermal conductivity.

The evolution of linear pumping comes to the end, and its concept to perfection, with the advent of the NEG thin film coatings developed at CERN in the last few years [6]. Thin films of getter materials, coated on the vacuum chamber inner walls by sputtering, have been shown to recover their gettering action, after exposure to ambient air, by "in situ" baking at temperatures as low as $180^{\circ} \mathrm{C}$ [7]. Besides removing all space and electric constraints, NEG coatings provide the largest possible pumping speed by transforming the vacuum chamber into a pump. Furthermore, the outgassing of the underlying surfaces and also the gas desorption induced by radiation and/or electron surface bombardment are strongly suppressed. This last benefit, which will not be discussed any further in this report, has been demonstrated both by dedicated experiments [8] and by real life operation [9] in existing machines.

To keep XHV under control, it must be possible to measure it. The evolution and the present state of the art of XHV measurements are reviewed in Section 2 of this report, while the main characteristics of NEG pumping 
are summarized in Section 3. Finally, a few examples of XHV conditions achieved by NEG pumping will be illustrated in Section 4.

\section{XHV PRESSURE MEASUREMENTS}

The main low pressure limitation of the ionization gauge, since its early days, was due to photo-emission of electrons from the ion collector under the effect of soft Xrays produced by the electron bombardment of the grid. Although the drastic reduction in collector area introduced by Bayard and Alpert (1950) [10] provided a decrease of this X-ray current $\left(\mathrm{I}_{\mathrm{x}}\right)$ as well as the corresponding pressure $\left(\mathrm{P}_{\mathrm{x}}\right)$ by two or three orders of magnitude, Alpert himself remarked some years later (1958) that it might be not possible to reduce $\mathrm{P}_{\mathrm{x}}$ indefinitely by reducing the collector wire diameter, because "there may be a critical size of the wire below which the probability of collecting ions goes down as rapidly as does the X-ray effect" [11].

This statement was confirmed by experimental results obtained with both open-ended and closed grids [11]. Some years later (1965) Comsa [12] came theoretically to the conclusion that the low pressure limit of the BayardAlpert gauge (BAG) should remain constant when reducing the collector wire diameter below $0.1 \mathrm{~mm}$, because the reduction of $\mathrm{I}_{\mathrm{x}}$ might be accompanied by an equal reduction of sensitivity. This conclusion was based on the fact that the tangential component of the speed of the ions produced inside the grid would prevent a fraction of them from colliding with the ion collector when first approaching it. These ions could not subsequently be collected, independently of the number of their orbits around the collector, if the radial electrostatic field present in the grid volume is ideally perfect. The number of "noncollectable" ions would increase proportionally to the reciprocal of the collector diameter below about $0.1 \mathrm{~mm}-$ hence the constant low pressure limit [12]. However, a systematic investigation carried out on a BAG with openended grid structure (Groszkowski, 1965 [13]) provided results in contradiction to this statement. In fact, a collector diameter reduction from 100 to $8 \mu \mathrm{m}$ produced a decrease in sensitivity of only a factor of 5 instead of the expected 12.5. Furthermore, already in 1961, Van Oostrom had shown that a $\mathrm{P}_{\mathrm{x}}$ lower than $10^{-12}$ Torr could be achieved with collector diameters as small as $4 \mu \mathrm{m}$ [14]. Schutze and Stork (1962) [15] also came to the conclusion that $\mathrm{P}_{\mathrm{x}}$ values as low as $5 \times 10^{-12}$ Torr are possible when using a collector of $40 \mu \mathrm{m}$ diameter and $10 \mathrm{~mm}$ length.

A few years later the BAG was practically abandoned for low pressure applications upon the appearance of gauges with collector external to the grid and providing $\mathrm{P}_{\mathrm{x}}$ below $10^{-12}$ Torr (Clay and Melfi, 1966 [16]; Redhead, 1966 [17]; Groszkowski, 1966 [18]; Helmer an Hayward, 1966 [19]).

The ISR interest for the low pressure behavior of the BAG originated from the need of replacing about 400 pressure gauges of this type which were installed on the ISR vacuum system. These gauges became obsolete as a result of the vacuum improvement (from $10^{-10}$ Torr to $\cong$ $5 \times 10^{-12}$ Torr, $\mathrm{N}_{2}$ equivalent) which has been found necessary to inhibit pressure instabilities in presence of intense proton beams. The main specifications for the new gauges were to provide pressure measurements in the pico-Torr range being compatible with the existing power supplies, which had been designed for modulated BAGs. This type of gauge has, therefore, been investigated in details providing the following main conclusions [20]:

- The electric field perturbation inside the grid produced by the filament voltage is sufficient to allow the collection of the otherwise "non collectable" ions, provided that the grid is properly closed at its extremities. In this case the ion collector diameter may be decreased to $20 \mu \mathrm{m}$ without any appreciable decrease of collection efficiency (i.e. gauge sensitivity);

- The X-ray limitation, lowered by decreasing the collector diameter, may be lowered even further by enlarging the grid diameter. Doing so, the electron path inside the grid is lengthened and more ions are produced per electron, providing the additional bonus of an increased sensitivity.

By enlarging the grid diameter from $25 \mathrm{~mm}$ to $35 \mathrm{~mm}$ and adopting a collector diameter of $50 \mu \mathrm{m}$, the BAG developed for the ISR is able to measure pressures in the XHV range with an accuracy (disregarding the errors on sensitivity calibration) of $2 \times 10^{-13}$ Torr. Time constraints did not allow a more exhaustive optimization to be carried out. Even better results could be obtained by further enlarging the grid (possibly up to $50 \mathrm{~mm}$ diameter) and reducing the collector diameter (to about $15 \mu \mathrm{m}$ ).

Although quite low, the average ISR pressure was not low enough for the intersection regions, where protons were brought to collision. Here pressures at least an order of magnitude lower (i.e. in the range of $10^{-13}$ Torr) were requested to reduce the proton-gas background to the particle detectors.

In order to measure these pressures, another development has been undertaken, aimed at improving the "Helmer gauge" (HG) produced by Varian [19]. In this gauge the ion produced inside the grid are extracted and driven to the external ion collector by electrostatic fields. The collector is enclosed in a box, to shield it from Xrays, and protected by a grid negatively biased to suppress photoelectrons. The low pressure limitation of the HG in its original version was $2 \times 10^{-12}$ Torr, due to tungsten vapors produced by the hot cathode [21]. An order of magnitude improvement was easily achieved by thoria coating the tungsten cathode so as to reduce its operating temperature. A further important improvement resulted from adopting the same larger grid diameter as for the BAGs [22]. Thanks to these changes, this improved gauge (IHG) is now able to measure pressures in the $10^{-14}$ Torr range with an accuracy of $3 \times 10^{-14}$ Torr [22].

As for the BAG, also the IHG could be further improved (see Section 4), but no requests have been formulated since the ISR era to justify a new development. Therefore the pressure gauges developed 
for the ISR about 30 years ago are still the best instruments for XHV measurements.

\section{NEG PUMPING}

Rare gases and light hydrocarbons do not react chemically with NEGs, either in the bulk or in the thin film form, and therefore they are not pumped.

The pumping probability (or sticking factor) for the other gases varies from 4 to $8 \times 10^{-1}$ for $\mathrm{CO}$ and from 0.6 to $3 \times 10^{-2}$ for $\mathrm{H}_{2}$, corresponding to specific pumping speeds of about 5 to $10 \mathrm{ls}^{-1} \mathrm{~cm}^{-2}$ for $\mathrm{CO}$ and 0.25 to $1.3 \mathrm{ls}$ ${ }^{1} \mathrm{~cm}^{-2}$ for $\mathrm{H}_{2}$. The spread of these values, relative to perfectly clean NEG surfaces, is a consequence of surface roughness. Rough surfaces enhance the pumping probability by favoring multiple gas-surface interactions.

When a NEG is operated at room temperature, only $\mathrm{H}_{2}$ diffuses inside the getter, while all the other gases remain on the surface, resulting in a progressive pumping speed decrease. This feature, illustrated for the NEG pump of LEP in Fig. 1, has been quantitatively described as a consequence of the progressive surface saturation along the microporosities in the getter structure [23]. By growing very rough thin films, their surface capacity may reach that of bulk getters in spite of being thinner by about a factor of 50 (Fig. 2).

In real life, not pure gases but gas mixtures must be pumped. It has been found that all NEGs behave similarly in this respect. Since $\mathrm{H}_{2}$ does not remain on the surface, its pumping does not affect the pumping of other gases, while $\mathrm{CO}$ inhibits it completely. An intermediate behavior is observed for $\mathrm{N}_{2}$, which practically does not change the pumping of $\mathrm{CO}$ and reduces that of $\mathrm{H}_{2}$ by $30 \%$ only after full saturation [24].

The oxide layer, formed on the getter surface upon air exposure, may be removed by heating under vacuum (activation). During activation the oxygen moves out from the surface and gets dissolved into the getter volume, which therefore gets progressively loaded with oxygen at each air exposure-activation cycle (aging). The process results, as all aging processes, in a life limitation. Longer lives may be provided by getter materials presenting a higher solubility limit for oxygen at the activation temperature. For a given getter, the death may be postponed by progressively increasing the temperature of activation. For instance, a TiZrV film, $5 \mu \mathrm{m}$ thick only, may undergo 50 aging cycles with a final decrease to $30 \%$ of its initial pumping speed for $\mathrm{H}_{2}$, provided that the temperature of activation is progressively increased from $180^{\circ} \mathrm{C}$ to $350^{\circ} \mathrm{C}$.

The widest difference among NEG materials concerns their temperature of activation, which ranges from the $750{ }^{\circ} \mathrm{C}$ of the $\mathrm{ZrAl}$ alloy and the $350^{\circ} \mathrm{C}$ of the $\mathrm{ZrVFe}$ alloy commercially available in the form of strips from SAES Getters [24], to the $180^{\circ} \mathrm{C}$ of the TiZrV thin film coating developed at CERN [7]. It should be noted that only thin film NEG coatings may present very low activation temperatures without being pyroforic, thanks to the good thermal contact with the substrate, which limits the temperature increase due to oxidation during exposure to air.

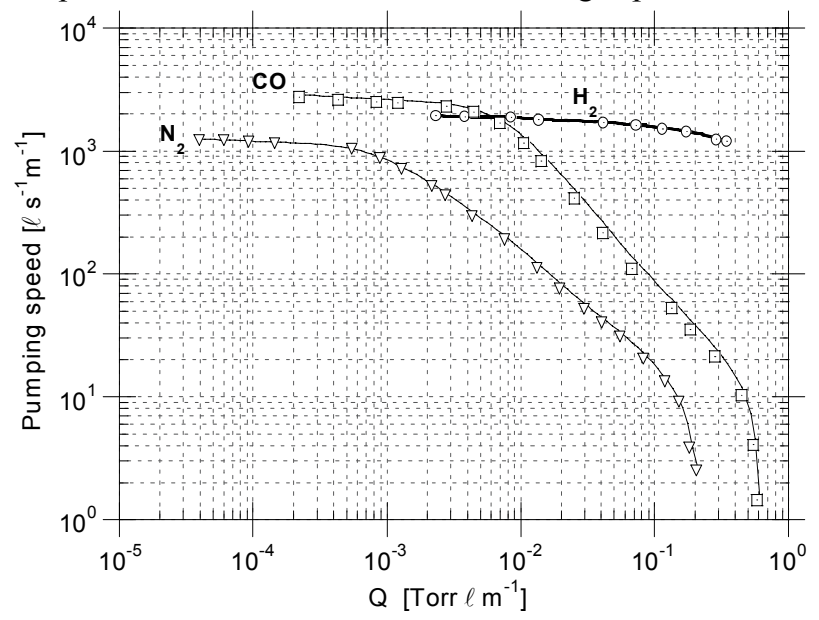

Fig. 1 Variation of the measured pumping speed for $\mathrm{H}_{2}$ (且), $\mathrm{CO}(\odot)$, and $\mathrm{N}_{2}(\nabla)$ as a function of adsorbed quantities $(\mathrm{Q})$ for the same sample of St 101 NEG strip. Full lines are a guide to the eye. The $\mathrm{H}_{2}$ injection rate is $6 \times 10^{-4}$ Torr $1 \mathrm{~s}^{-1} \mathrm{~m}^{-1}$.

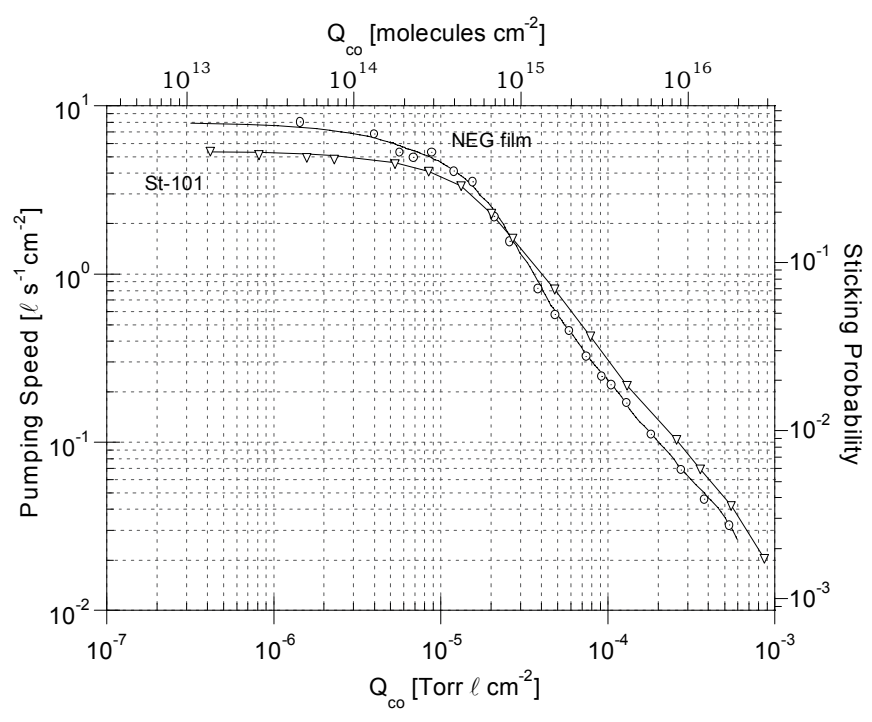

Fig. 2 Comparison of the surface capacity for $\mathrm{CO}$ of a very rough NEG thin film and of the St 101 NEG strip.

\section{ULTIMATE PRESSURES: Fact or fiction?}

Legitimate doubts may arise when measurements of $10^{-13}$ Torr or even lower are reported. To be plausible, these measurements must be complemented by various types of cross checks, of which a few examples are given below.

For vacuum system of simple geometry, it is good practice to compare the measured ultimate pressure with the calculated ratio of outgassing rate to pumping speed, quantities which may be measured independently at higher pressures. In some cases, like for NEG strips, the total pumping speed may be evaluated from short sample 
measurements. Whenever possible, the applied pumping speed may be varied in a controlled way, or a part of the system may be cooled with liquid $\mathrm{N}_{2}$ to provide a well defined pumping speed for $\mathrm{Ar}$ and $\mathrm{CH}_{4}$, gases not pumped by NEGs. This latter practice is particularly useful because mass spectrometers cannot be used in some cases due to their important outgassing.

These procedures are illustrated by means of the following examples:

\subsection{The LEP dipole chambers}

The main pumping for these chambers, $12 \mathrm{~m}$ long, was provided by a NEG strip (of the type St 101 produced by SAES Getters) of $11 \mathrm{~m}$ length and $30 \mathrm{~mm}$ width. Auxiliary pumping for $\mathrm{CH}_{4}$ and rare gases was supplied by a Sputter-Ion pump of $30 \mathrm{ls}^{-1}$ nominal speed. The added speed for $\mathrm{H}_{2}$ of this pump was marginal (less than $1 \%)$.

In the absence of unforeseen events, the ultimate pressure achieved after baking was about $2 \times 10^{-12}$ Torr, while an ultimate $\mathrm{H}_{2}$ pressure of about $2 \times 10^{-13}$ Torr was expected on the ground of the measured outgassing rate of the chamber and of the NEG strip pumping speed.

According to the mass spectrometer, enough $\mathrm{CH}_{4}$ and Ar were present to justify the measured total pressure, but unfortunately the information on the $\mathrm{H}_{2}$ pressure was missing due to a very large $\mathrm{H}_{2}$ parasitic peak in the instrument. This missing information was particularly important because of a widespread belief that below $10^{-11}$ Torr the NEG pump would rather outgas $\mathrm{H}_{2}$ than pump it [26].

In order to clarify the situation, six additional SputterIon pumps of the same size as the one originally installed have been added to a LEP chamber, and the total ultimate pressure has been measured switching those pumps on one by one. The result indicates that, with all pumps in operation, the total pressure approached the expected $\mathrm{H}_{2}$ pressure [27]. The IHG measurements were found to agree closely with those of the ISR type BAGs, showing the good performance of these gauges in the $10^{-13}$ Torr range [27].

\section{2 "Total"NEG pump}

A $3 \mathrm{~m}$ long stainless steel chamber of $160 \mathrm{~mm}$ diameter has been equipped with a NEG pump obtained by winding a NEG strip on a stainless steel cage of the same length as the chamber and of similar diameter. The strip used to produce this pumping liner was of the type St 707, produced by SAES Getters, which may be activated passively during bakeout at about $350^{\circ} \mathrm{C}$.

Also in this case the outgassing rate of the chamber had been measured before inserting the NEG pump, of which the pumping speed has been evaluated from short sample measurements. The ultimate pressure, measured by the IHG, was about $3.5 \times 10^{-14}$ Torr, while no more than $1 \times 10^{-14}$ Torr was expected. This discrepancy has been interpreted as an indication that the measuring limits of this gauge had been reached [28].

\subsection{Thin film NEG coated chambers}

Depending on sample geometry, many different methods have been adopted to evaluate the quality of a NEG coating. For long vacuum chambers, both the ultimate pressure after activation and the sticking factors for various gases are measured on a dedicated system equipped with Sputter-Ion pump and Titanium Sublimation pump liquid $\mathrm{N}_{2}$ cooled [29]. The system may reach an ultimate pressure in the low $10^{-}$ ${ }^{12}$ Torr range. The chamber to be tested is connected at one extremity to this system via an orifice of $25 \mathrm{ls}^{-1}$ conductance for $\mathrm{H}_{2}$, which has the function of minimizing the gas exchange with the main vacuum system, and at the other extremity is equipped with an IHG which measures the ultimate pressure. The sticking factor is obtained by Monte Carlo simulation from the measured pressure drop across the chamber while injecting gas from one end. Usually $\mathrm{H}_{2}$ is used for this operation because it is more sensitive to surface cleanliness, it does not change the surface conditions during the measurement and provides more accurate information thanks to its low sticking factor.

Various chambers $2 \mathrm{~m}$ long and of diameter ranging form $40 \mathrm{~mm}$ to $100 \mathrm{~mm}$, coated with a TiZrV film, have been measured. While the sticking factors were found to be quite reproducible for comparable activation conditions, the ultimate pressures were spread over one order of magnitude, being lower for larger diameters [29].

Since a chamber of larger diameter provides a higher pumping speed at the location of the gauge, it has been concluded that the IHG gauge was measuring its own outgassing. This conclusion is confirmed by Fig. 3, where the ultimate pressures are plotted versus the chamber pumping speed [29].

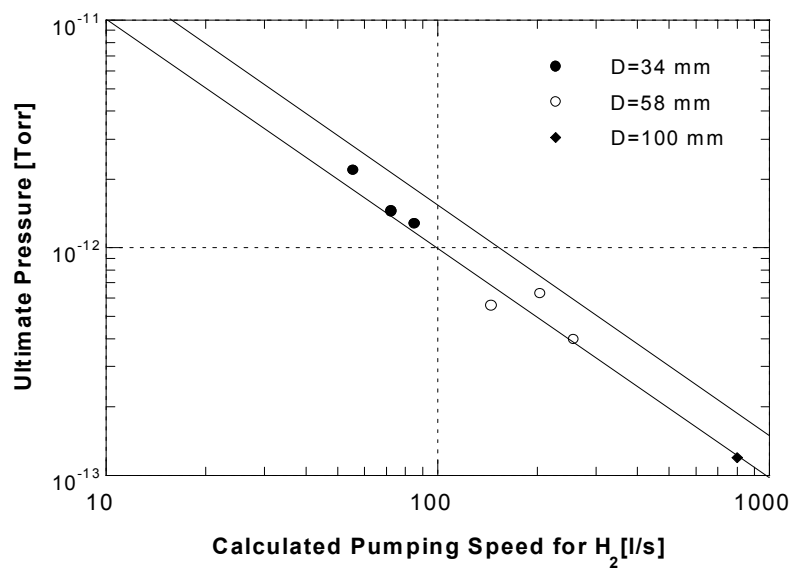

Fig. 3. Variation of the measured ultimate pressures as a function of the $\mathrm{H}_{2}$ pumping speed provided by the NEG coated chambers at the location of the measuring gauge. The ultimate pressures are measured by the same IHG gauge on $2 \mathrm{~m}$ long chambers of different diameter $\mathrm{D}$ after different activation procedures. The full lines represent the upper and lower limits of the contribution due to the measured gauge degassing. The uncertainty on the gauge sensitivity is $20 \%$. 
It may be observed that also the result described in par. 4.2 is compatible with this conclusion. The IHG provides accurate measurements in the $10^{-14}$ Torr range, but it perturbs the pressure to be measured.

\section{CONCLUSIONS}

Vacuum technology has evolved during the last 30 years to provide better operating conditions for particle accelerators. The pumping has moved closer to the vacuum chamber, where the gases to be pumped are produced, resulting in very large pumping speeds, and, in the case of NEG thin film coating, also in lower outgassing rates. As a by-product, in the absence of beam induced outgassing, pressures in the XHV range are now routinely achievable inside vacuum systems of any length and cross section.

The limits for the quest of low pressures are set by the measuring capabilities rather than by the pumps or the vacuum system construction materials. Furthermore, the limit seems to be a consequence of the outgassing of the measuring instrument rather than of its intrinsic measuring possibilities.

If this is the case, a further progress could be possible if the IHG gauge outgassing could be reduced. Gas analysis carried out on a dedicated system indicates that this outgassing is not of thermal origin. In this case, the spectrum would be dominated by $\mathrm{H}_{2}$, while in reality a large presence of $\mathrm{CO}$ is observed, which is typical for electron or radiation stimulated desorption.

The bias voltages applied to the gauge electrodes prevent the ionizing electrons from reaching the surrounding vacuum chamber walls. On the other hand, it is common practice to "degas" the gauge electrodes while baking the vacuum system. During this operation the grid is heavily bombarded by electrons (typically of $700 \mathrm{eV}$ energy and $50 \mathrm{~mA}$ intensity) and gets red hot. Therefore it is unlikely that enough contamination remains on the grid to justify the outgassing observed.

It seems more plausible to assume that the gas is desorbed from the surrounding surfaces by the soft X-rays produced by the electrons impinging on the gauge grid. In this case much lower outgassing rate should be obtained by NEG coating all the surfaces around the gauge and the collector shielding box. Coating also the internal surfaces of the box and the deflecting electrodes would provide the additional advantage of reducing the background which limits the low pressure performance of the IHG gauge [22].

If this reasoning is correct, these improvements could lead to measuring pressures in the $10^{-15}$ Torr range.

\section{REFERENCES}

[1] E. Fischer, Proc. 3rd All-Union National Conf. on Particle Accelerators, Isdatel'stvo Nauka, Moscow, (1973)
[2] J.S. Kouptsidis, Proc. 7th Int. Vac. Congr. 1, (1977) 341

[3] C. Benvenuti, Nucl. Instr. Meth. Phys. Res. 205, (1983) 391

[4] H.P. Reinhard, Proc. 9th Int. Vac. Congr., (1983) 273

[5] C. Benvenuti and P. Chiggiato, Vacuum, 44, (1993) 511

[6] C. Benvenuti, P. Chiggiato, F. Cicoira and Y. L'Aminot, J. Vac. Sci. Technol. A 16 (1), (1998) 148

[7] C. Benvenuti, P. Chiggiato, P. Costa Pinto, A. Escudeiro Santana, T. Hedley, A. Mongelluzzo, V. Ruzinov and I. Wevers, Vacuum, 60, (2001) 57

[8] P. Chiggiato and R. Kersevan, Vacuum, 60, (2001) 67

[9] R. Kersevan, Proc. of the EPAC 2000 Conf. (Vienna)

[10] R. T. Bayard and D. Alpert, Rev. Sci, Instrum., 21, (1950) 571

[11] D. Alpert, Encyclopedia of Physics, edited by Flugge, Springer-Verlag, Berlin Vol. 12 (1958) 624

[12] G. Comsa, J. Appl. Phys., 37, 2, (1966) 554

[13] J. Groszkowski, Bull. Acad. Polon. Sci. Ser. Sci. Techn., 13, (1965) 2

[14] A. Van Oostrom, Trans. AVS Vac. Symp., (1961) 8443

[15] H.J. Schutze and F. Stork, Trans. AVS Vac. Symp., (1962) 9431

[16] F.P. Clay and L.T. Melfi, J. Vac. Sci. Technol., 3, (1966) 167

[17] P.A. Redhead, J. Vac. Sci. Technol., $\underline{3}$, (1966) 173

[18] J. Groszkowski, Bull. Acad. Polon. Sci., 14, (1966) 1023

[19] J.C. Helmer and W.H. Hayward, Rev. Sci. Instrum., 37, (1966) 1652

[20] C. Benvenuti and M. Hauer, Nucl. Instr. Meth., 140, (1977) 453

[21] B. Angerth and Z. Hulek, J. Vac. Sci. Technol., 11, (1974) 461

[22] C. Benvenuti and M. Hauer, Le Vide, les Couches Minces, 201, (1981) 199

[23] C. Benvenuti and F. Francia, J. Vac. Sci. Technol. A, 6, no. 4, (1988) 2528

[24] C. Benvenuti and F. Francia, J. Vac. Sci. Technol. A, 8 , no. 5 , (1990) 3864

[25] C. Boffito, B. Ferrario, P. della Porta and L. Rosai, J. Vac. Sci. Technol., 18, no. 4, (1981) 1117

[26] J.H. Singleton, J. Vac. Sci. Technol. A, 8, no. 1, (1971) 275

[27] C. Benvenuti, J.P. Bojon, P. Chiggiato and G. Losch, Vacuum, 44, (5-7), (1993) 507

[28] C. Benvenuti and P. Chiggiato, Vacuum, 44, (1993) 511

[29] C. Benvenuti, A. Escudeiro Santana and V. Ruzinov, Vacuum, 60, (2001) 279 